\title{
Difusión Social de la Ciencia en los Medios de Comunicación Extremeños
}

\author{
Irene Ruiz Jiménez
}

María J. Reyes Barragán

Ma Victoria Nuño Moral

Recibido: 15 de noviembre de 2014

Aceptado: 8 de diciembre de 2014

\section{Resumen}

En los últimos años organismos nacionales e instituciones científicas abogan por la cultura y difusión de la ciencia, siguiendo la legislación en materia de ciencia y tecnología. Este trabajo analiza la presencia de la ciencia en los medios de comunicación, centrándose en los dos periódicos regionales más importantes de Extremadura: el diario Hoy y El Periódico Extremadura. La dimensiones cuantitativas analizadas son (1) Producción de noticias científicas; (2) Evaluación de noticias científicas en el periodo objeto de estudio, 2011; (3) Noticias científicas recuperadas por descriptores de búsqueda y (4) Clasificación de las noticias científicas por campos temáticos de la ANEP (Agencia Nacional de Evaluación y Prospectiva de España). Los resultados muestran la escasa repercusión que tienen las noticias científicas en los periódicos Hoy y Periódico de Extremadura, lo que refleja la limitada cultura científica en la sociedad extremeña.

\section{Pallabras clave}

Ciencia y Tecnología, Cultura científica, Periódicos digitales, Diario Hoy, El Periódico Extremadura

\section{Social Spread of Science in the Mass Media from Extremadura}

\section{Abstract}

In recent years, national agencies and scientific institutions advocate scientific culture and dissemination, following the legislation of science and technology. This paper analyzes the presence of science in the media, focusing on two major regional newspapers in Extremadura: the Hoy and El Periódico Extremadura. The quantitative dimensions analyzed are (1) Production of scientific news; (2) Evaluation of scientific news in the period under review, 2011; (3) Science news descriptors retrieved by search and (4) Classification of scientific news into the thematic fields of the ANEP (National Evaluation and Foresight of Spain). The results show the negligible impact of scientific news in the newspapers Hoy and Periódico de Extremadura, reflecting the limited scientific culture in the society of Extremadura

\section{Keywords}

Science and Technology, Scientific Culture, digital newspapers, Diario Hoy, El Periódico Extremadura

http://dx.doi.org/10.5209/rev CDMU.2014.v25.47478 


\section{INTRODUCCIÓN}

Difundir y fomentar la cultura científica es imprescindible en una sociedad abierta y participativa como la actual. Los medios de comunicación convencionales desempeñan un papel sustancial en la difusión de los avances científicos, ya que tienen un alto poder de influencia sobre una gran audiencia. La realización y difusión de noticias científicas requiere de periodistas especializados que cuenten con ayuda de expertos en las materias para aclarar, contextualizar o ampliar la información.

Hoy en día la mayoría de los periódicos, programas de televisión o revistas ofrecen secciones dedicadas a las novedades científicas. La difusión científica en los medios supone, como señala Moreno Castro (2004): "un esfuerzo por infundir conocimientos más profundo de la ciencia en esta sociedad, para que los ciudadanos puedan tomar decisiones cotidiana en este campo (...) El periodismo científico debe estar encaminado a que los ciudadanos entiendan los problemas tecno-científicos que les afectan con la menor ambigüedad posible".

Esta misma autora señala que "a los investigadores y grupos de investigación les interesa difundir sus trabajos ante los ciudadanos a través de los medios de comunicación convirtiéndose el debate mediático en un garante para obtener más ayuda económica (pública o privada)". Aunque la estimación de los datos no son muy bondadosos, según el Instituto Nacional de Estadística, los gastos totales de las ayudas económicas destinadas a I+D en 2011 descendieron un 2'8\% con respecto al año 2010. En el caso de Extremadura, el descenso fue del 5'2\%. El gasto de personal en los dos casos fue del 3'1\% y del $7 \%$ respectivamente.

A este respecto, sobre la divulgación social de la ciencia, la Ley de Ciencia de 2011 recoge como objeto de ésta: "Establecer el marco para el fomento de la investigación científica y técnica y sus instrumentos de coordinación general, con el fin de contribuir a la generación, difusión y transferencia del conocimiento para resolver los problemas esenciales de la sociedad". Esta idea se afianza en los puntos j y I del artículo 2 de dicha ley:

j. "Impulsar la cultura científica, tecnológica e innovadora a través de la educación, la formación y la divulgación en todos los sectores y en el conjunto de la sociedad".

I. “Promover la participación activa de los ciudadanos en materia de investigación, desarrollo e innovación, y el reconocimiento social de la ciencia a través de la formación científica de la sociedad y la divulgación científica y tecnológica, así como el reconocimiento de la actividad innovadora y empresarial".

En su artículo 37, regula lo relativo a la difusión de acceso abierto:

2. "El personal de investigación cuya actividad investigadora esté financiada mayoritariamente con fondos de los Presupuestos Generales del Estado hará pública una versión digital de la versión final de los contenidos que le hayan sido aceptados para publicación en publicaciones de investigación seriadas o periódicas, tan pronto como resulte posible, pero no más tarde de doce meses después de la fecha oficial de publicación". 
3. "La versión electrónica se hará pública en repositorios de acceso abierto reconocidos en el campo de conocimiento en el que se ha desarrollado la investigación, o en repositorios institucionales de acceso abierto".

5. "El Ministerio de Ciencia e Innovación facilitará el acceso centralizado a los repositorios, y su conexión con iniciativas similares nacionales e internacionales".

Por último, la Ley en el artículo 38 en el apartado 2.a propone: "Mejorar la formación científica e innovadora de la sociedad, al objeto de que todas las personas puedan en todo momento tener criterio propio sobre las modificaciones que tienen lugar en su entorno natural y tecnológico".

En la Comunidad Autónoma de Extremadura, la Ley 10/2010, de la Ciencia, la Tecnología y la Innovación, en su artículo 4, fija sus fines abriendo camino a la divulgación científica en dos apartados:

c. "Contribuir a la difusión y el aprovechamiento en la Comunidad Autónoma de Extremadura de los resultados de la investigación científica y técnica, el desarrollo tecnológico y la innovación, especialmente los generados en la Región".

ñ. "Promover la cultura científica y tecnológica entre los ciudadanos de la Comunidad Autónoma de Extremadura, estimulando la difusión de la ciencia, la tecnología y la innovación, así como divulgando la actividad científica como herramienta de conexión entre la ciencia y la sociedad y entre la ciencia y el tejido empresarial".

El artículo 5., apartado e., expone como uno de los principios informadores: "La participación y divulgación al conjunto de los ciudadanos del papel de la ciencia, la tecnología y la innovación en sus vidas cotidianas". El artículo 7 de la presente Ley se centra en la sensibilización, divulgación y participación sobre las actividades del Sistema Extremeño de Ciencia, Tecnología e Innovación, haciendo referencia en el apartado 3, al papel de los medios de comunicación en la difusión científica: "La Junta de Extremadura a través de los medios de comunicación velará por la difusión y comunicación de la I+D+l”. En el artículo 10 se determinan las funciones de la consejería competente, recalcándose en el apartado I lo referente a la divulgación científica: "Contribuir a que la población extremeña valore el conocimiento como un valor en sí mismo y asuma la importancia de la investigación. Con tal fin, fomentará la divulgación de los avances del conocimiento y la comprensión de sus principios básicos a través de los medios de difusión más adecuados".

Así mismo, establece que tanto la Universidad de Extremadura como las Fundaciones y Academias, tengan entre sus finalidades la divulgación del conocimiento en cualquiera de sus formas.

La divulgación de la ciencia no es fácil ya que entraña cierta dificultad de expresión o de entendimiento, lo que puede distorsionar la actividad científica. Sabadell Melado (2003) en su artículo "Ciencia, Sociedad y Medios de Comunicación", hace hincapié en diferentes niveles de comunicación de la ciencia: la popularización y la divulgación. Mientras que la popularización tiene como finalidad interesar al lector, la segunda se dirige a informar a un lector ya interesado en el tema.

La ciencia como conocimiento cierto, exacto y razonado de las cosas por sus principios y causas, ha sido y es algo inherente a la actividad humana y a la sociedad. Pero hasta el siglo XX, no se ha producido el nacimiento de la ciencia moderna y su crecimiento exponencial (Price, 1963). La ciencia contemporánea es 
esencialmente abierta y libre para abordar todos los problemas nuevos, en la medida en que se encuentren métodos para tratarlos (Piaget, 1988). De hecho, en el último siglo, hemos asistido a grandes descubrimientos, que anteriormente se producían en grandes intervalos de tiempo. El progreso científico y los cambios acaecidos conllevan a una modificación de las características de la propia ciencia y su relación con la sociedad. Los nuevos métodos de producción requieren inevitablemente transformaciones sociales para poder adaptarlos con éxito, y su progreso se debe, en gran parte, a la importancia de la ciencia en su contexto social (Bernal, 1989).

Gracias al avance científico-tecnológico y a la proliferación del uso de la web, en el s. XXI la información tiene un papel crucial en todos los ámbitos de la vida. Los medios de comunicación, como señalan Villas Tinoco y Montiel Torres (2000) "le han dado a los sistemas de información un alcance mundial: los sistemas de difusión por satélite traspasan la fronteras y las redes de comunicación (con Internet como paradigma), conectan países y continentes. Asentada en ellos, la globalización se esparce por todos los campos, no sólo como extensión geográfica sino como incremento en el uso de la información por el público en general: para sus actividades como consumidores (de bienes o de servicios públicos o privados), como ciudadanos (en su relación con las instituciones o con otros ciudadanos) o en las actividades formativas o culturales (incluidos el ocio y el entretenimiento)".

En el caso de Extremadura el sector informativo atraviesa momentos de incertidumbre al tener que adaptarse a los continuos cambios tecnológicos.

El periódico Hoy sacó el 1 de enero de 1933 el primer número en papel. Durante años fue evolucionando: (1) en 1987 informatizó todo el proceso de producción con una red de ordenadores Macintosh; (2) en 1994, el periódico sacó su primera impresión en color; (3) en 1995 se realizó un trabajo de digitalización de textos y fotos, para que los periodistas pudiesen reutilizar esa información en posteriores artículos desde sus ordenadores, conectados a la red; (4) en 2006, el periódico saca su versión digital (http://www.hoy.es/). El primer documento que se rescata de la hemeroteca digital del periódico data del 2 de abril del 2006.

El Periódico Extremadura, con su sede central en Cáceres, se fundó en abril de 1923. En 1998 pasa a formar parte del grupo Zeta, "el cual, por la difusión de sus publicaciones y por su enorme influencia social, se sitúa entre los primeros grupos editoriales españoles". En este mismo año, empieza la impresión a color. En el año 1999, como consecuencia del avance tecnológico, según Villar (2013), responsable de la edición digital del periódico, el rotativo amplía su oferta a través de Internet, creando el portal de El Periódico Extremadura (http://www.elperiodicoextremadura.com/index.php). No obstante, el primer documento que se recupera en su hemeroteca digital data del 15 de noviembre de 2002. "Se trataba de una web 1.0 y en ella sólo aparecían los contenidos de la edición impresa. La participación de nuestros lectores se centraba en la mera observación. A lo largo del tiempo, el diario optó por demandar la colaboración de los lectores, creando una comunidad virtual y abandonando la rigidez de un sitio estático para beneficiarse de la versatilidad que la web 2.0 ofrece a sus usuarios, contribuyendo con ello a que nuestros usuarios participen en el proceso creativo del diario".

En este contexto, el trabajo que se presenta intenta dar respuesta al grado de implicación de los medios de comunicación en la divulgación social de la ciencia, analizando cuantitativamente la difusión de las noticias 
científicas por clases temáticas en los periódicos de Extremadura: Hoy y El Periódico Extremadura en su versión digital en el año 2011 (www.hoy.es y http://www.elperiodicoextremadura.com/).

\section{MÉTODO Y DATOS}

Para conseguir el objetivo planteado anteriormente, y fijada la temporalidad del estudio, se determinaron los descriptores más significativos para realizar las búsquedas en los periódicos digitales, los cuales nos permitirían recuperar las noticias científicas. Estos fueron los siguientes: Investigación, Investigadores, I+D, Innovación, Ciencia, Política científica, Actividad científica, y Universidad de Extremadura. Para su desarrollo, se creó una base de datos con objeto de almacenar las noticias recuperadas.

La clasificación temática de las noticias recuperadas en ambos periódicos siguió la clasificación de la ciencia que hace la Agencia Nacional de Evaluación y Prospectiva (ANEP). Esta clasificación es una de las más extendidas en la comunidad científica española y sigue unos patrones internacionales. Por ello, se optó por esta nomenclatura. Adicionalmente se barajó también la clasificación de la UNESCO.

\begin{tabular}{l}
\hline Fisica y Ciencias del Espacio \\
Ciencias de la Tierra \\
Matematicas \\
Quimicas \\
Biologia Molecular, Celular y Genetica \\
Medicina \\
Fisiologia y Farmacologia \\
Biologia Vegetal, Animal y Ecologia \\
Agricultura \\
Ganaderia y Pesca \\
Ciencia y Tecnologia de los Alimentos \\
Ciencia y Tecnologia de los Materiales \\
Ciencia De La Computacion y Tecnologias Informaticas \\
Ingenieria Civil y Arquitectonica \\
Ingenieria Electrica, Electronica y Automatica \\
Ingenieria Mecanica Naval y Aeronautica \\
Tecnologia Quimica \\
Tecnologia Electronica y de las Comunicaciones \\
\hline Psicologia y Educacion \\
Ciencias Sociales \\
\hline Derecho \\
Economia \\
\hline Filologia y Filosofia \\
Historia y Arte \\
I+D \\
\hline Inversion \\
\hline General \\
\hline Iacion tematica de Ia Agencia Nacionalde Evaluacion y Prospetive
\end{tabular}

Tabla 1. Clasificación temática de la Agencia Nacional de Evaluación y Prospectiva (ANEP).

La clasificación temática que se muestra en la tabla 1, se complementó con tres apartados temáticos más: I+D, Inversión y General. La inclusión de estos apartados pretende dar cabida a ciertos artículos que no se podían incluir en la nomenclatura de la ANEP. Además, resultaba interesante saber la importancia social que 
se le daba a la investigación y a la inversión en I+D. El apartado General que se incluyó, hace referencia a los artículos que trataban diversos contenidos como: jornadas, simposios, premios científicos, artículos sobre la situación de la ciencia, becas de investigación y artículos de opinión científica.

Una vez sentadas las bases, se realizaron búsquedas en las hemerotecas digitales de dichos periódicos. Estas se llevaron a cabo por cada descriptor en el año establecido, revisando cada artículo rescatado por la aplicación. Cuando un artículo estaba relacionado con la ciencia se introducía toda la información necesaria en la base de datos. Algunos descriptores compartían artículos y para evitar la duplicidad, con cada nuevo descriptor que se usaba había que comprobar si el título de los artículos que se introducían estaban o no en la base de datos.

Una vez introducidos los datos, se pasó a ejecutar las consultas en la base de datos. La dimensión cuantitativa se realizó desde cuatro vertientes: (1) Producción de noticias científicas; (2) Evaluación de noticias científicas en el periodo objeto de estudio, 2011; (3) Noticias científicas recuperadas por descriptores de búsqueda y (4) Clasificación de las noticias científicas por campos temáticos de la ANEP.

\section{RESULTADOS Y DISCUSIÓN}

La base de datos cuenta con 400 noticias rescatadas sobre ciencia en los periódicos extremeños, de las cuales 202 son de El Periódico Extremadura (PEx) y 198 del diario Hoy.

1) Producción de noticias científicas

Las tasas de noticias de ciencia rescatadas, respecto al total de noticias publicadas en el año 2011 en cada periódico (Figura 1), muestran la cruda realidad de la ciencia en la sociedad. Tan sólo el 0’23\% de las 86.915 noticias publicadas en el Hoy y el 0’324\% de 62.347 noticias publicadas en EI PEx están relacionados con la ciencia.

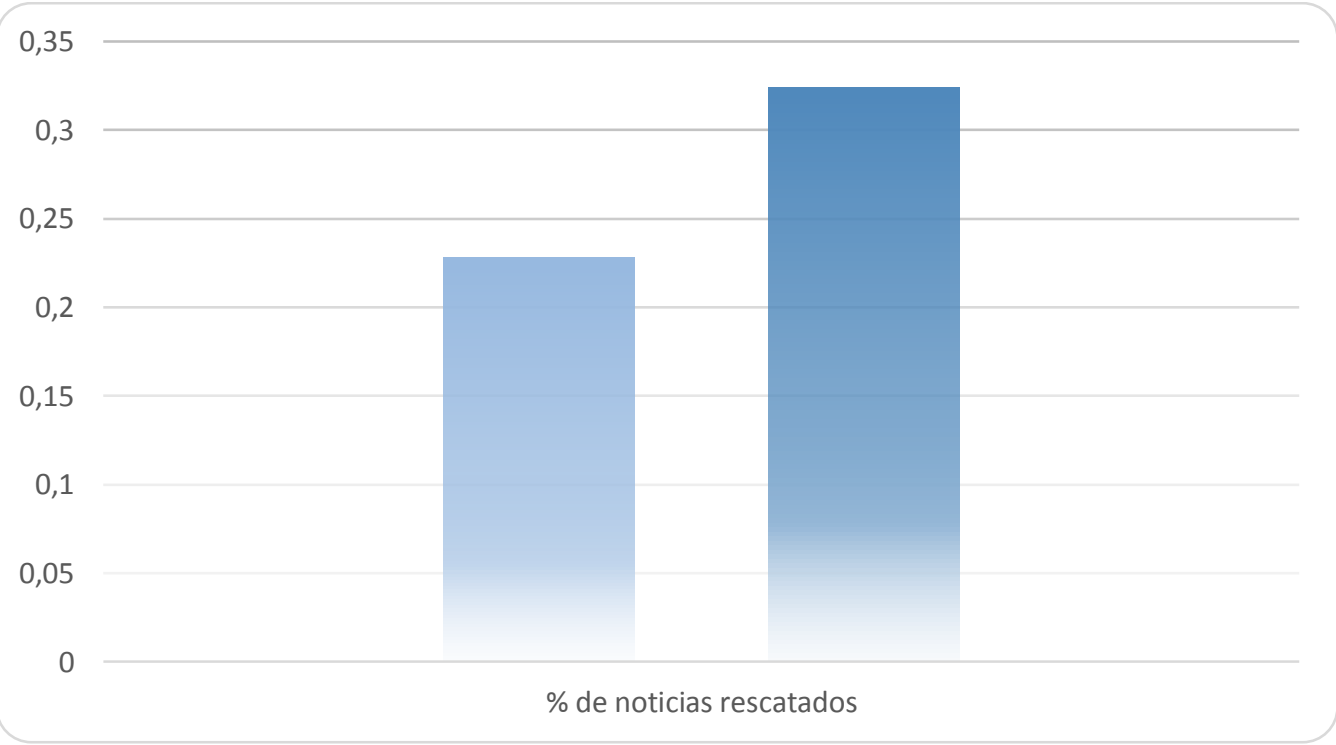

Figura 1. Tasa de noticias de ciencia rescatadas, respecto al total de noticias publicadas en el año 2011. 
Esta muestra pone de manifiesto que no se le presta la importancia que requiere la divulgación social de la ciencia en los medios de comunicación. Por el contrario, las noticias centradas en política, economía y deportes son las que cubren la mayor parte de las noticias en los periódicos extremeños.

\section{2) Noticias rescatadas por mes de publicación}

La evolución de la producción en los meses del año objeto de estudio, muestra que las tasas de noticias sobre ciencia rescatadas y publicadas en una determinada fecha, respecto del total de noticias recuperadas, indican que no hay una constante en la publicación; es decir, no se aprecia un patrón de comportamiento en las fechas de publicación de las noticias científicas obtenidas (Figura 2).

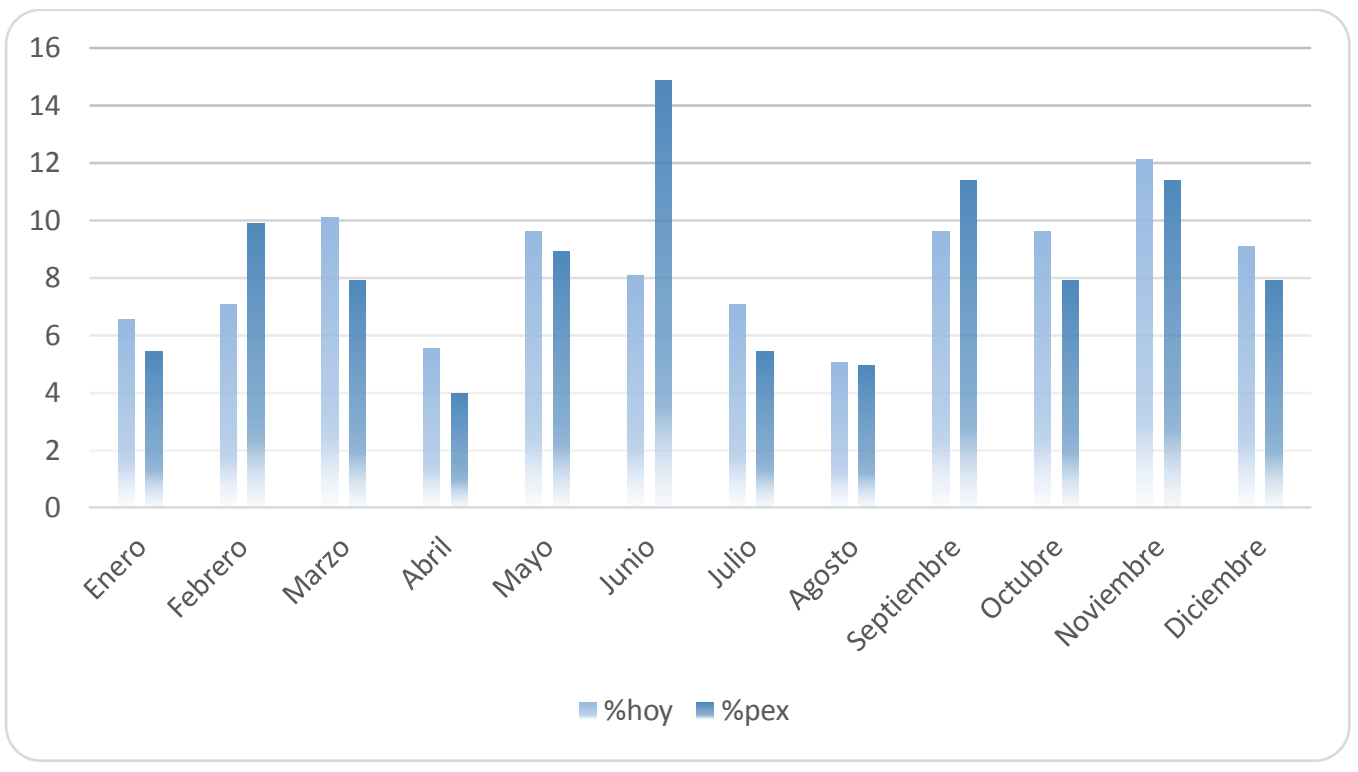

Figura 2. Tasa de noticias de ciencia rescatadas y publicadas respecto al total de noticias, 2011

\section{3) Noticias recuperadas por Descriptores}

El total de noticias recuperadas con los descriptores utilizados en las hemerotecas digitales se muestra en la tabla 2. Como puede observarse los valores son muy altos, debido a que el motor de búsqueda de las hemerotecas funciona buscando el descriptor en el texto de los artículos. Esto hace que se recuperen muchos más noticias de las que se necesitan, produciendo ruido documental, ya que se obtienen noticias de otras temáticas muy diferentes.

Las noticias científicas de cada periódico y para cada descriptor se representan en la tabla 3. La comparativa de ambas tablas pone de manifiesto una significativa diferencia entre ambas. Es de reseñar que en el caso del descriptor " $I+D$ " no se recuperara ninguna noticia en la hemeroteca digital del periódico Hoy, aunque nos consta que sí aparecía en otras noticias rescatadas del mismo periódico. Esto se debe a que el motor de búsqueda de este diario no admite el signo "+" como parte del texto de búsqueda, sino que lo utiliza como un operador booleano. 


\begin{tabular}{|l|l|l|}
\hline DESCRIPTORES & HOY & PEX \\
\hline Investigación & 4850 & 2316 \\
\hline Investigadores & 1164 & 472 \\
\hline I+D & 0 & 150 \\
\hline Innovación & 937 & 824 \\
\hline Ciencia & 1359 & 453 \\
\hline Política científica & 4 & 14 \\
\hline Actividad científica & 2 & 24 \\
\hline Universidad de Extremadura & 1794 & 950 \\
\hline
\end{tabular}

Tabla 2. Noticias recuperadas en los periódicos

\begin{tabular}{|l|l|l|l|}
\hline DESCRIPTORES & HOY & PEX & TOTAL \\
\hline Investigación & 136 & 150 & 286 \\
\hline Investigadores & 88 & 102 & 190 \\
\hline I+D & 13 & 33 & 46 \\
\hline Innovación & 33 & 56 & 89 \\
\hline Ciencia & 115 & 68 & 183 \\
\hline Política científica & 0 & 1 & 1 \\
\hline Actividad científica & 2 & 1 & 3 \\
\hline Universidad de Extremadura & 43 & 71 & 114 \\
& & & \\
\hline
\end{tabular}

Tabla 3. Noticias rescatadas de los periódicos

En la figura 3 se muestra la alta tasa de noticias del periódico hoy, rescatadas con el descriptor "actividad científica". El 100\% significa que el total de noticias recuperadas por el motor de búsqueda de este periódico son de ciencia, algo que como contraposición, no ocurre con el pex que sólo cuenta con el $4 \%$ de las noticias recuperadas.

Otro dato importante a señalar en esta figura es que en el resto de descriptores, la tasa más alta de noticias relacionadas con la ciencia siempre se presenta en el pex, siendo los descriptores "investigadores" e " $i+d$ " los que tienen una tasa mayor con un $21^{\prime} 6 \%$ y un $22 \%$, respectivamente. El resto de descriptores tienen una tasa de noticias sobre ciencia que ronda el $10 \%$ respecto al total de noticias que recupera el sistema de las hemerotecas digitales. Es destacable que la tasa es mayor en los descriptores en el pex que en el hoy, lo que significa que el sistema de el pex recupera una tasa más alta de noticias relacionadas con la ciencia y que por tanto, tienen menos ruido documental.

El descriptor "I+D" en el hoy tiene una tasa del 0\%; esto ocurre porque el motor de búsqueda no recuperó ninguna noticia. Sin embargo, en el caso del descriptor "política científica", la tasa es del $0 \%$ porque se recuperaron y rescataron 0 noticias.

Por el contrario, las tasas respecto al total de noticias rescatadas son muy diferentes, como se muestra en la figura 4. Hay que destacar que la mayoría de los descriptores presentan una tasa mayor de noticias en el pex 
que en el diario hoy. Esto se debe a que se han rescatado más noticias con el primero. La única excepción se encuentra en los descriptores "actividad científica" y "ciencia". El primero no supone una gran diferencia entre los dos periódicos ya que sus tasas son del 1'01\% para el hoy y 0'49 para el pex. En el caso del descriptor "ciencia" la diferencia no se ajusta tanto; sus tasas son de 58 '08\% para el diario hoy y de 33 ' $66 \%$ en el pex.

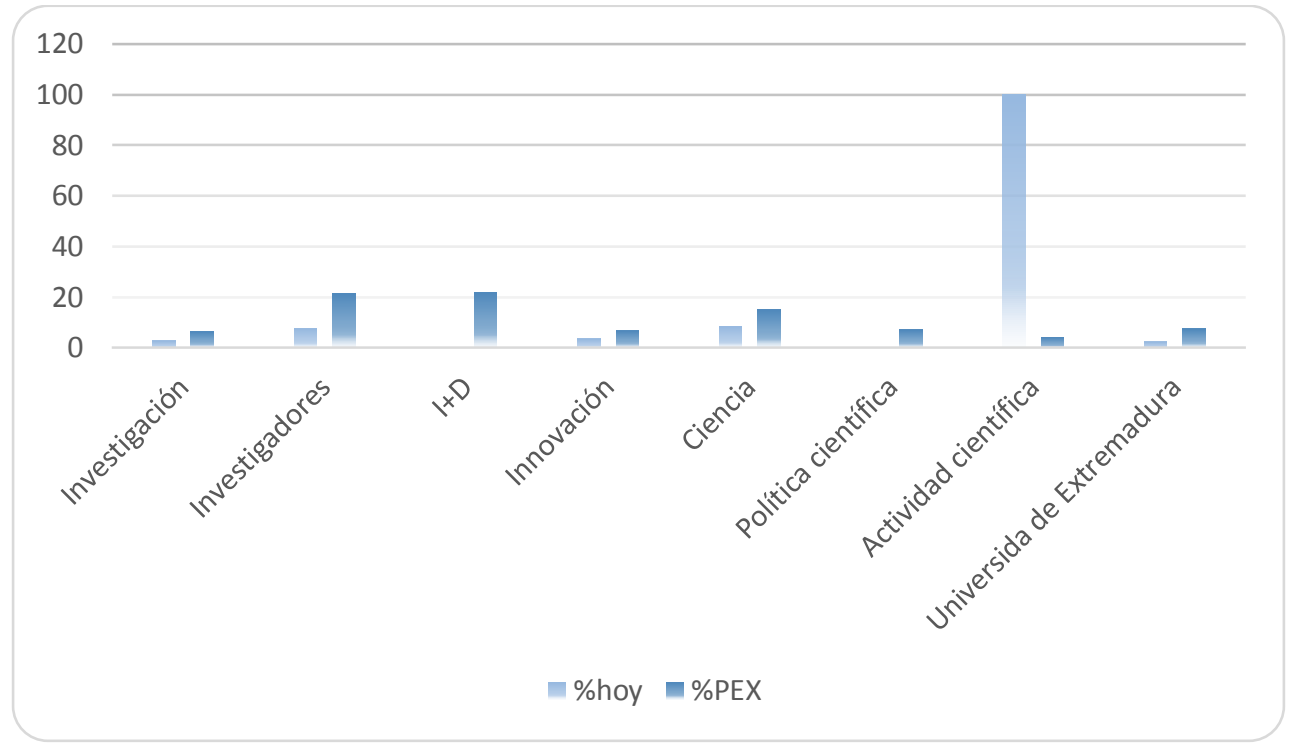

Figura 3. Tasa de noticias rescatadas con unos descriptores, respecto al total de noticias recuperadas en las hemerotecas de los periódicos.

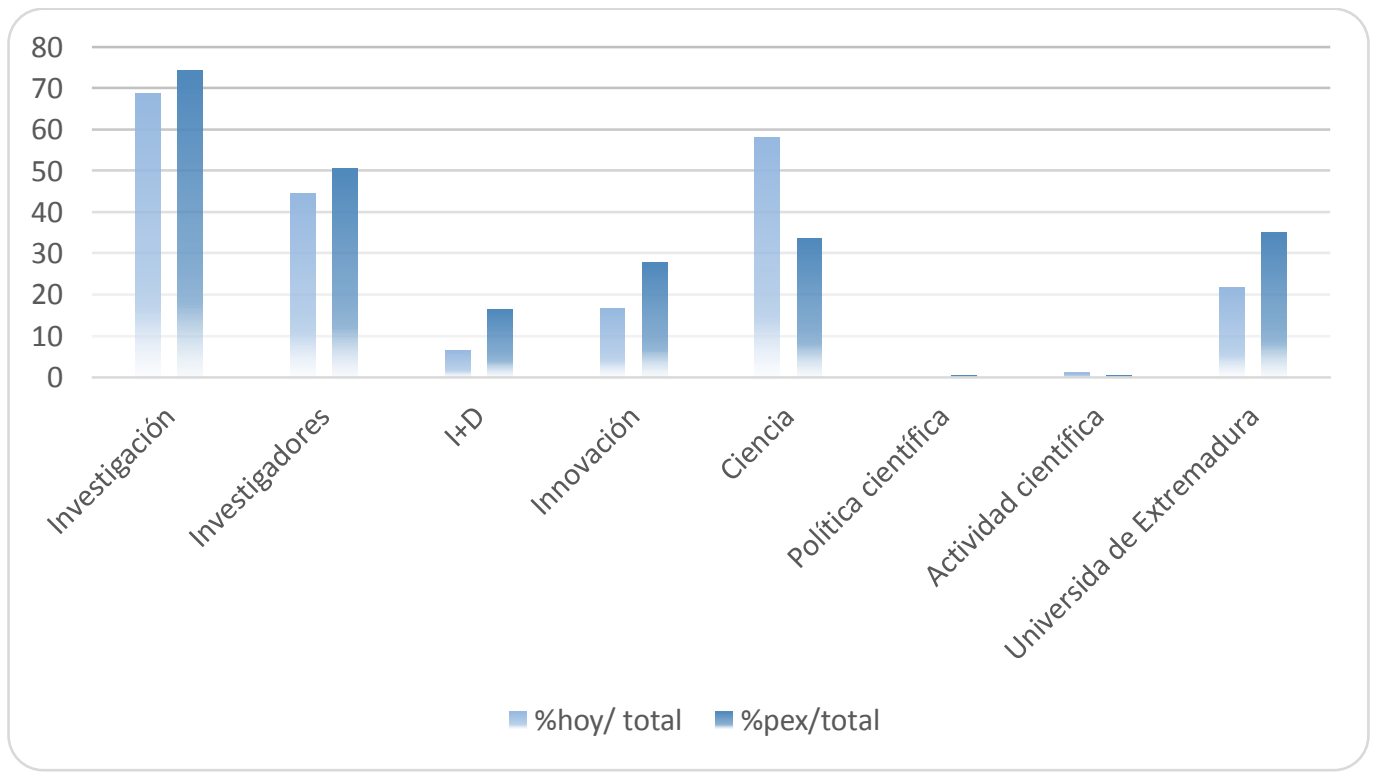

Figura 4. Tasa de noticias rescatadas con unos descriptores, respecto al total de noticias rescatadas. 
Los descriptores con mayor tasa de noticias son, en orden decreciente, "investigación", "investigadores" y "ciencia". Los que tienen una tasa menor son "política científica" y "actividad científica". Estos niveles ya se hacían evidentes con los datos brutos (tabla 3) y cuando se recuperaban las noticias de los periódicos.

Las temáticas con más noticias son: Medicina, General y Física y Ciencias del espacio. Los descriptores que más noticias rescatados con las diferentes temáticas son: Investigación, Investigadores, Ciencia y Universidad de Extremadura. Siendo Política científica y Actividad científica los que menos noticias rescataron de las diferentes temáticas.

4) Clasificación Temática de las Noticias Científicas

El análisis de las temáticas analiza las noticias que se publican en cada periódico, de acuerdo a la clasificación de la ANEP. En la tabla 4 podemos observar que hay 9 temáticas que no tienen registrado ninguna noticia. 


\begin{tabular}{|l|l|l|l|}
\hline TEMÁTICAS & HOY & PEX & TOT. \\
\hline MEDICINA & 52 & 61 & 113 \\
\hline FISICA Y CC ES & 53 & 14 & 67 \\
\hline GENERAL & 15 & 47 & 62 \\
\hline HISTR. Y ARTE & 21 & 7 & 28 \\
\hline I+D & 6 & 16 & 22 \\
\hline QUIMICAS & 10 & 6 & 16 \\
\hline BIOL. MOLEC. & 3 & 11 & 14 \\
\hline FISIO Y FARMA & 5 & 9 & 14 \\
\hline INGEN. ELEC. Y AUTM. & 11 & 2 & 13 \\
\hline INVERSION & 2 & 9 & 11 \\
\hline TEC. ELEC. COMUNICAC. & 3 & 7 & 10 \\
\hline CC SS & 7 & 1 & 8 \\
\hline CC TIERRA & 4 & 3 & 7 \\
\hline BIOL VEG. & 1 & 5 & 6 \\
\hline AGRICULTURA & 1 & 4 & 5 \\
\hline MATEMATICAS & 1 & & 1 \\
\hline INGEN. MEC. NAV. Y AER & 1 & & 1 \\
\hline TEC. QUIMICA & 1 & & 1 \\
\hline GANAD. Y PES. & & & \\
\hline CCY TEC. ALIM & & & \\
\hline CCY TEC. MAT. & & & \\
\hline CC COMP. & & & \\
\hline INGEN. CIVIL Y ARQ & & & \\
\hline PSICO. Y EDU. & & & \\
\hline DERECHO & & & \\
\hline ECONOMIA & & & \\
\hline FILOL. Y FILOS. & & \\
\hline \multicolumn{1}{|c|}{ TEDa } & & \\
\hline
\end{tabular}

Tabla 4. Número de noticias de una temática

Las tasas de noticias rescatadas que pertenecen a cada temática concreta se exponen en la figura 5; en ella se han seleccionado las temáticas con más de una noticia rescatada de los periódicos. 


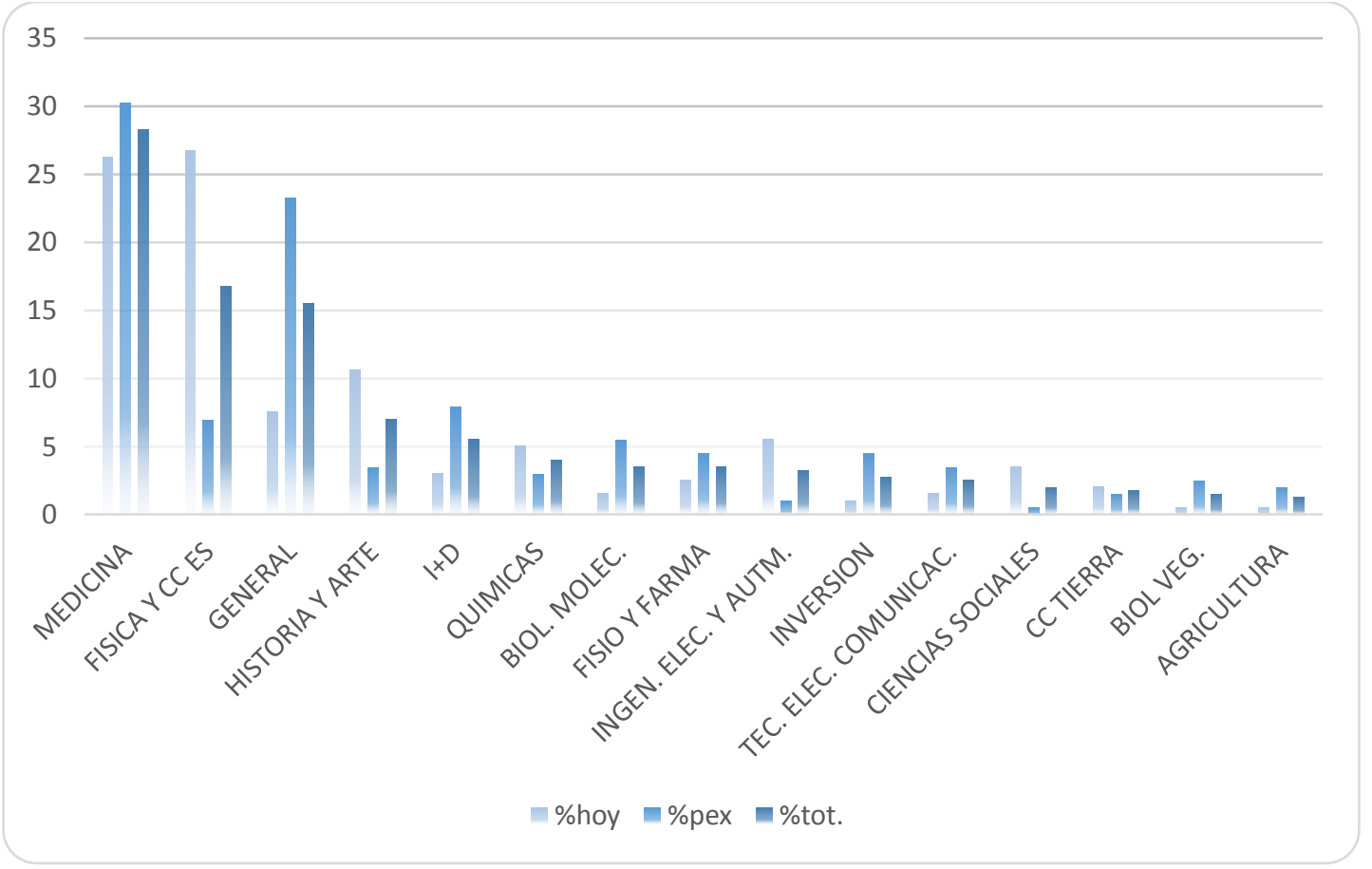

Figura 5. Tasa de noticias rescatadas de cada temática.

En la tabla 5 se observa como muchas de las temáticas no tiene datos, lo que se debe a que no se utilizaron en la base de datos en ningún momento. Algunas celdas en blanco corresponden a El PEx que, como se puede ver, publica más artículos pero utiliza menos temáticas que su homólogo Hoy.

Por su parte, este último publica en más temáticas que EI PEx. Las temáticas más destacadas son Física y Ciencias del espacio (26,8\%), Medicina (26,3\%), Historia y arte (10,6\%), artículos de Ciencia general $(7,58 \%)$, Ingeniería eléctrica, electrónica y automática $(5,56 \%)$ y Química $(5,05 \%)$. Las menos repetidas tienen unas tasas que oscilan entre 3,54\% y 0,51\%: Ciencias de la tierra, Matemáticas, Biología molecular, celular y genética, Fisiología y farmacología, Biología vegetal, animal y ecología, Agricultura, Ingeniería mecánica naval y aeronáutica, Tecnología química, Tecnología electrónica y de las comunicaciones, I+D e Inversión. Estas dos últimas tasas, son muy relevantes en el mundo científico; en el Hoy cuenta con un 3'03\% y un 1 '01\% respectivamente. Estos valores tan bajos podrían indicar el escaso esfuerzo que se hace en I+D en Extremadura y la escasa inversión en ciencia.

En el caso de EI PEx, la temática más productiva es Medicina con una tasa del 30,2\%; las noticias de Ciencia en general aumentan drásticamente hasta llegar al 23,3\%. Algo sorprendente ocurre con I+D, que supera a Física y Ciencias del espacio, teniendo el primero un 7,92\% y el segundo un 6,93\%. Es decir, Física y ciencias del espacio pasa a un cuarto lugar dando paso a I+D. Seguidos de estos, tenemos Biología molecular, celular y genética con un $5,45 \%$ de noticias recuperadas. 


\begin{tabular}{|l|l|l|l|}
\hline TEMÁTICAS & \% Hoy & \% PEx & \% Total \\
\hline MEDICINA & $\mathbf{2 6 , 2 6}$ & $\mathbf{3 0 , 2}$ & $\mathbf{2 8 , 2 5}$ \\
\hline FISICA Y CC ES & $\mathbf{2 6 , 7 7}$ & $\mathbf{6 , 9 3 1}$ & $\mathbf{1 6 , 7 5}$ \\
\hline GENERAL & $\mathbf{7 , 5 7 6}$ & $\mathbf{2 3 , 2 7}$ & $\mathbf{1 5 , 5}$ \\
\hline HISTORIA Y ARTE & $\mathbf{1 0 , 6 1}$ & $\mathbf{3 , 4 6 5}$ & $\mathbf{7}$ \\
\hline I+D & $\mathbf{3 , 0 3}$ & $\mathbf{7 , 9 2 1}$ & $\mathbf{5 , 5}$ \\
\hline QUIMICAS & 5,051 & $\mathbf{2 , 9 7}$ & $\mathbf{4}$ \\
\hline BIOL. MOLEC. & 1,515 & 5,446 & 3,5 \\
\hline FISIO Y FARMA & 2,525 & 4,455 & 3,5 \\
\hline INGEN. ELEC. Y AUTM. & 5,556 & 0,99 & 3,25 \\
\hline INVERSION & 1,01 & 4,455 & 2,75 \\
\hline TEC. ELEC. COMUNICAC. & 1,515 & 3,465 & 2,5 \\
\hline CIENCIAS SOCIALES & 3,535 & 0,495 & $\mathbf{2}$ \\
\hline CC TIERRA & 2,02 & 1,485 & 1,75 \\
\hline BIOL VEG. & 0,505 & 2,475 & 1,5 \\
\hline AGRICULTURA & 0,505 & 1,98 & 1,25 \\
\hline MATEMATICAS & 0,505 & & 0,25 \\
\hline INGEN. MEC. NAV. Y AER & 0,505 & & 0,25 \\
\hline TEC. QUIMICA & 0,505 & & 0,25 \\
\hline GANAD. Y PES. & & & \\
\hline CC Y TEC. ALIM & & & \\
\hline CC Y TEC. MAT. & & & \\
\hline CC COMP. TEC. INFORMAT. & & & \\
\hline INGEN. CIVIL Y ARQ & & & \\
\hline PSICO. Y EDU. & & & \\
\hline DERECHO & & & \\
\hline ECONOMIA & & & \\
\hline FILOL. Y FILOS. & & & \\
\hline & & & \\
\hline
\end{tabular}

Tabla 5. Tasa de noticias por temática, respecto al total de noticias rescatadas

Otra diferencia con el periódico Hoy es la tasa de las noticias sobre Inversión y de Fisiología y farmacología, que alcanzan el $4,46 \%$. No parece una cifra relevante, pero si se comparan ambos medios, se aprecia un gran aumento. El resto de temáticas tienen unas tasas que oscilan entre 3,47\% y 0,5\% en las siguientes temáticas: Ciencias de la tierra, Química, Biología vegetal, animal y ecología, Agricultura, Ingeniería eléctrica, electrónica y automática, Tecnología electrónica y las comunicaciones y Ciencias sociales.

Las tasas del total de noticias reflejan que Medicina sigue siendo la temática más utilizada con un 28,3\%, seguida de Física y ciencias del espacio con un $16,8 \%$ y de los noticias sobre Ciencia en general con un 15,5\%. Historia y Arte cuenta con un 7\%, superando el resultado de EI PEx, pero no el del Hoy. Caso contrario ocurre con I+D, que presenta una tasa de 5,5\% respecto al total. El resto de temáticas tienen unas tasas entre $4 \%$ y 0,25\% para: Matemáticas, Química, Biología molecular, celular y genética, Fisiología y farmacología, Biología vegetal, animal y ecología, Agricultura, Ingeniería eléctrica, electrónica y automática, Ingeniería mecánica naval y aeronáutica, Tecnología química, Tecnología electrónica y las comunicaciones, Ciencias sociales e Inversión. Las tasas del total de los periódicos varían compensando el déficit de un periódico u otro. 
El índice de actividad $^{1}$ para cada temática se muestra en la figura 6 . Este índice proporciona información sobre el volumen e impacto de las actividades de investigación, y pretende evidenciar las diferencias entre los campos del conocimiento. En este caso todos los índices son muy inferiores a 1, no llegando a superar el 0 ’30. Puesto que son resultados muy bajos y con muchos decimales se han seleccionado sólo los datos de las temáticas cuyo índice de actividad era superior a 0,1.

Como en casos anteriores, en esta gráfica analizaremos por separado cada serie. En el periódico Hoy, la mayoría de los índices superan a los de las otras series, excepto en contadas ocasiones. Las temáticas con el mayor índice de actividad son Física y ciencias del espacio con un 0,27, seguida de Medicina con un 0,26 e Historia y arte que cuenta con un índice del 0,10. El resto de índices cuentan con valores muy inferiores al 0,1 .

Los resultados de El PEX no son muy diferentes. Las cuatro temáticas con un índice más alto de esta serie son: Medicina con un 0,3 y artículos sobre Ciencia en general con 0,23 . El resto de índices presentan valores inferiores a 0,1.

Respecto al total de ambos periódicos, las temáticas que tienen un mayor índice de actividad son las siguientes: Medicina con un índice del 0,28, Física con 0,17 y artículos generales con 0,16. El resto de índices son muy inferiores al 0,1.

Como se aprecia, el esfuerzo de las temáticas es inferior al promedio, ya que todos los índices de actividad de estas temáticas son inferiores a 1.

$1 \quad$ Índice de actividad. El índice de actividad proporciona información sobre el volumen e impacto de las actividades de investigación. Este índice pretende evidenciar las diferencias entre los campos del conocimiento. Cuando el índice de actividad es superior a 1, la clase temática realiza un esfuerzo superior al esfuerzo que se realiza de forma promedio en los demás campos. Si es inferior a 1, el esfuerzo de ese campo es inferior al promedio del resto de los campo. Y si es igual que 1, el esfuerzo del campo es similar al promedio de todos los campos. En este caso todos los índices son muy inferiores a 1, no llegando a superar el 0’30. 


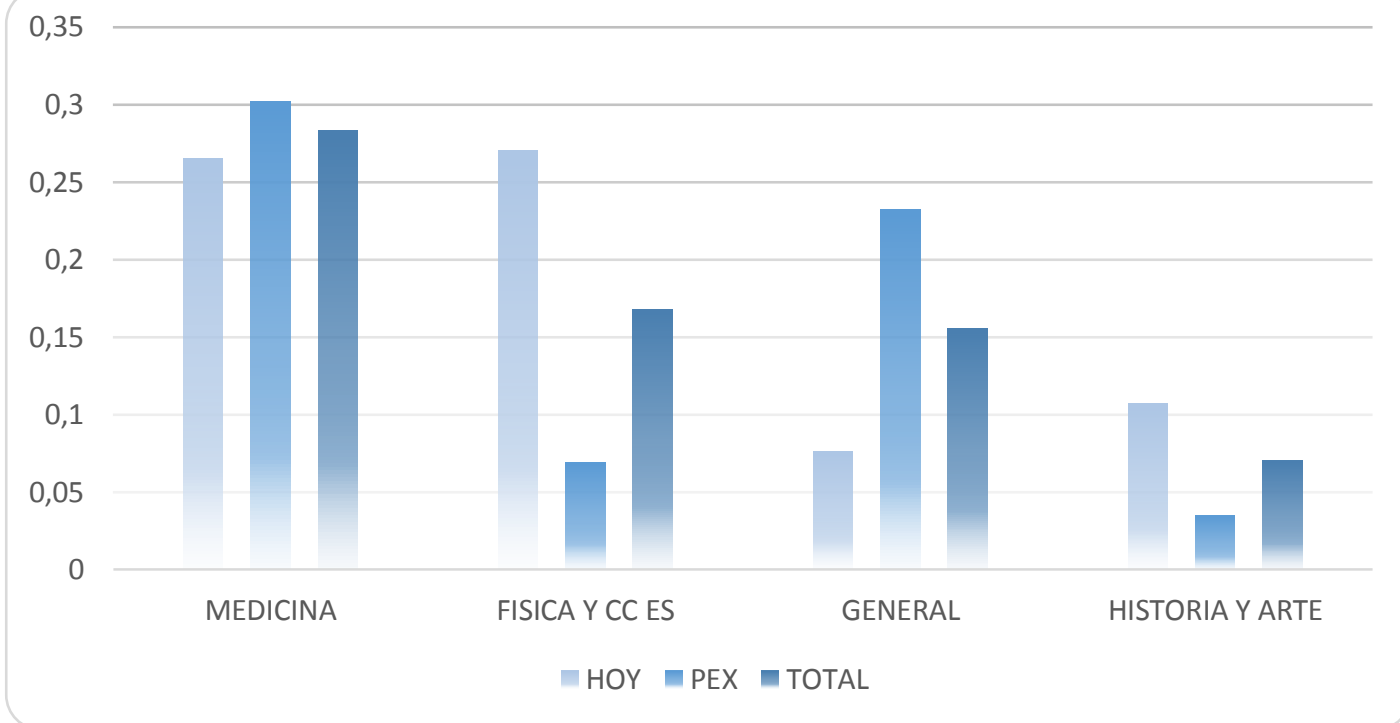

Figura 6 . Índice de actividad de cada temática para cada periódico y el total.

\section{CONCLUSIONES}

En primer lugar, y atendiendo al estado de la cuestión de este estudio, se puede decir que los valores que arrojan las tasas de noticias de ciencia recuperados, no evidencian un interés social por las noticias sobre ciencia. Este hecho puede ser debido a que el análisis se ha realizado en periódicos regionales, lo que podría incluso desvincularlos aún más de la función de divulgar la actividad científica a los ciudadanos.

Resulta llamativa la diferencia entre el número de noticias recuperadas por las hemerotecas de los periódicos y el número de noticias rescatadas de estas. Es decir, los motores de búsqueda de las hemerotecas recuperaban un cierto número de noticias con cada descriptor, pero sólo una ínfima parte de estos estaban realmente relacionados con la ciencia. Esto significa que hay un problema en los motores de búsqueda en las hemerotecas los periódicos, ya que presentan mucho ruido documental.

La tasa de artículos sobre ciencia rescatados, no supera el 0’4\% en ninguno de los dos periódicos. Sin duda, es una cifra desalentadora que conlleva a una infravaloración de la actividad científica y su divulgación.

Es significativo que el descriptor "Política científica" tenga una tasa tan baja respecto a los otros descriptores. Esto pone en evidencia que las instituciones públicas no prestan demasiado interés en difundir las políticas públicas en materia de Ciencia y Tecnología.

No se ha detectado un incremento de la producción en los diferentes meses del año objeto de estudio. Se puede presuponer que el ligero descenso de artículos que se produce en los meses de verano (julio y agosto) se debe a que los autores disfrutan de su periodo vacacional, disminuyendo la actividad divulgadora de la información en general y de esta temática en particular. 
Las temáticas con más actividad son, en nuestra opinión, las que suscitan un mayor interés entre los lectores de los periódicos. Estas temáticas por lo general son: Medicina, Física y ciencias del espacio, artículos generales e Historia y arte.

Si lo comparamos con los resultados obtenidos en la distribución reflejada en el estudio llevado a cabo en el diario La Voz de Galicia, en general, los resultados serían muy similares. En el citado estudio las dos temáticas con más actividad son también Salud y Espacio (como las denominan en este estudio) con un 19\% y $13 \%$ respectivamente. El resto de temáticas no coincide en sus tasas con las que aquí se establecen; esas serían: Medio ambiente 13\%, Biología 11\%, Química 11\%, Nuevas tecnologías 9\%, Genética 7\%, Arqueología $7 \%$, Ciencias cognitivas $7 \%$ y otros $3 \%$. Los resultados, excepto en las dos temáticas con más actividad, son algo diferentes a los presentados en este estudio. En esta ocasión, es conveniente señalar que el 3\% englobado bajo el indicador "Otros" en el estudio gallego, abarcaría, en nuestro caso, una amplia parte del trabajo ya que quedarían englobadas muchas de las temáticas que han aparecido en el caso extremeño (López, Xosé, Otero, María Luisa y Calvo, Ma Dolores, 2013).

Finalmente, destaca la falta de atención a las temáticas I+D e Inversión, que tienen unas tasas muy bajas. Esto resulta llamativo más aún con los crecientes recortes que la Administración General y Autonómica está realizando últimamente en el campo de la investigación científica. Por ello, aún teniendo tasas más altas que en otras temáticas, en nuestra opinión son muy bajos, ya que no se hacen eco de los problemas actuales de la investigación científica y de la situación de la ciencia en España y en la Comunidad de Extremadura. Esto es un inconveniente, porque si a la sociedad no se le informa de este grave problema, nunca se le llegará a dar la importancia que este ámbito del conocimiento se merece.

Por último, siguiendo la opinión de Juan Tena en su artículo "La ciencia desapercibida", existe una falta verdadera de información científica y tecnológica por escasez de espacios en los medios de comunicación y de periodistas capacitados. Los medios deben tomar conciencia social y brindar un espacio donde se le dé a todas las ramas del conocimiento, la importancia que realmente tienen. Todo ello, con el único propósito de que la ciencia alcance una mayor relevancia, valorando su inherente perspectiva cultural y su impacto social y económico. 


\section{BIBLIOGRAFÍA}

Bernal, J. D. (1989). Historia social de la ciencia (6ª ed.). Barcelona: Península, 2 vol. p. 17.

El Periódico de Extremadura. Historia de El Periódico de Extremadura [Consultado: 23-11-2013], desde <http://www.elperiodicoextremadura.com/info/historia.php>

España. Ley 14/2011, de 1 de junio, de la Ciencia, la Tecnología y la Innovación. Boletín Oficial del Estado, 2 de junio de 2011, núm. 131, pp. 54387 - pp. 54455.

Extremadura. Ley 10/2010, de 16 de noviembre, de la Ciencia, la Tecnología y la Innovación de Extremadura. Diario Oficial de Extremadura, 19 de noviembre de 2010, núm. 223, pp. 27194 - pp. 27245.

Instituto Nacional de Estadística (2012). Estadística Sobre Actividades en I+D [Consultado el 17/04/2013], desde <http://www.ine.es/prensa/np747.pdf>

López, Xosé; Otero, María Luisa; Calvo, María Dolores (2010). La divulgación de la ciencia en el diario "La voz de Galicia". En: AE-IC. Comunicación y Desarrollo en la Era Digital: Congreso Internacional AE-IC. (Málaga 3-5 Febrero 2010).

Ministerio de Economía y Competitividad. Agencia Nacional de Evaluación y Prospectiva (ANEP) (2013).

[Consultado: 31-10-2013], desde

<http://www.idi.mineco.gob.es/portal/site/MICINN/menuitem.26172fcf4eb029fa6ec7da6901432ea0/?vgnextoi d=3d5167b99490f310VgnVCM1000001d04140aRCRD>.

Moreno Castro, Carolina (2004). La información científico técnica y la formación democrática de los ciudadanos. En: La divulgación del conocimiento en la sociedad de la información (pp. 349-359). Santiago de Compostela: Escola Galega de Administración Pública

Piaget, Jean (1988). Sájese et illusions de la philosophie. Paris: Presses Universitaires de France, p. 52

Price, D. J. S. (1973). Hacia una ciencia de la ciencia. Barcelona: Ariel. Trad. de little science, big science, Columbia, 1963.

Sabadell Melado, Miguel Ángel (2003). Ciencia, sociedad y medios de comunicación. Física y Sociedad, 14, 35-39.

Tena, Juan (2003). La Ciencia Desapercibida. CTS: Revista Iberoamericana De Ciencia, Tecnología y Sociedad, 1, (1), pp. 189-196.

Villas Tinoco, Siro; Montiel Torres, Francisca (2000). Historia, ciencia y tecnología. Málaga: Universidad de Málaga. 